\title{
TFD: TELECOM FRAUD DETECTION USING CONSOLIDATED WEIGHTED REPUTATION ALGORITHM
}

\author{
J. Deepa Anbarasi, Ph.D, Research Scholar, Avinashilingam Institute for Home Science and Higher Education for Women, \\ Coimbatore-43.
}

Dr. V. Radha, Professor, Department of Computer Science, Avinashilingam Institute for Home Science and Higher Education for Women, Coimbatore-43.

\begin{abstract}
Noisy phone calls are aggravating and distracting, as well as frustrating. They may be classed as 'nuisance', 'emergency', 'random', and 'unsolicited' calls. Users have no inherent privileges on the internet; rather, their personalities are produced without any arrangement or evidence of involvement. It costs the U.S. communications company $\$ 8$ billion per year to avoid call spam on the phone grid. Between January 2014 and June 2018, the FTC (Federal Trade Commission) received over 22 million reports of fraudulent and illegal telemarketing calls. Nowadays, the mobile network is used to issue automatic phone calls such as robocalls. Since it operates on text, we struggle with the following: What tactics and methods do we use to combat spam? Telephone TFD (Telecom Fraud Detection) here is discussed first. Concerning spam, we advanced our proposal by proposing a targeted traffic detection using a single weighted credibility algorithm with appropriate weighting criteria.
\end{abstract}

Keywords: TelecomSpam, TFS, CDR, CWRA, VoIP

\section{Introduction}

Our mobile devices are dispensing an enormous volume of mobility and behavior info. On the one hand, however, mobile terminals can be closely monitored by telecom providers. However, as the cost of Internet access and Smartphone falls, mobile apps and social networks gain popularity, they become able to use geodata in a crossreferenced manner. Specifically, services such as Twitter, Flickr, or Foursquare, may provide us details about people and their activities in a sense that is described as being [3]

Ability issues are occurring in heavily developed cities. Several access network programs are ongoing. On the other side, we agree that, over a CDR Dataset, there are limitations in integrating them both. First, it may have a time chart, but it does not specify specifically when the network may be used. This is a central part of the operator's way of thought. Used, such as how many settings, when they should be added, and the ability to set each capacity. The second issue is that large-scale CDR selection delineates global patterns, but it disregards local differences [4].

Bogus or missing request headers can reduce the effectiveness of the feature. If the caller's phone number (captured from caller ID or Automatic Number Identification Service) were previously on a block list, it would automatically decline the call Callers from all other phone numbers are permitted, excluding calls from international numbers [9]. Spam blocks may be used to blacklist phone numbers suspected of sending spam messages, which blocks calls to the terminal without disrupting the user. ID Blocking is only placed in effect once the phone number is applied to the registry, but not with other phone numbers in which the caller wants to enter. Thus, it's simple for other callers to use [10]. We only allow white listed phone numbers in the telephone caller ID function. Otherwise, calls are not authorized. It may be used to remove spam phone numbers commonly recognized, but the receiver would not be informed of the non-trusted numbers. It doesn't take much investment, and it is simple to introduce. There are plenty of features available on modern smart phones. Checker ID analysis looks for bizarre caller ID patterns, such as nonworking area code, toll-free code, and pattern matching, to discover whether the caller is a spammer. Finding Caller ID Anomalies takes relatively few computing power and is therefore cheap and simple, so it's commonly used in call blocking applications.

This article is arranged as seen here. Section II presents previous studies focused on the analysis of CDRs. We introduced our framework in section III, detailing each of its components and operation. We present the discussion in section IV, and finally, conclude the paper in section V.

\section{Background Study}

V. A. Balasubramaniyan, et al. [1] the CallRank scheme that aims to spot spammers based on call length CallRank is used to predict success over time, encouraging genuine callers to be made and defeating spammers. All and all, we will quickly introduce new subscribers while stopping scammers from upsetting the remainder of the email list.

H. Sengar, et al. [2] to counter the possible voice-spam hazard, two complementary and realistic steps were employed. According to Mahodis distance, the first scheme aims to recognize suspicious activity at the subscriber 
stage. Instead of detecting each call length, the second method employed call durations as a proxy to aggregate the results. A misbehaving party of subscribers will be shown an anti-spam alert.

Cecaj, A. et al. [3] we were able to conclude that it's possible to reliably classify people who are likely to be the same through several databases, with our success. While our method is still being refined, the findings have proved quite encouraging. Additionally, it will allow for the correct re-assignment of users' activities with more details and added functionality.

Srihari, V. et al. [5] Emerging pattern in telecommunications is VoIP, which transmits voice and data over the packet-switched IP networks. SPIT is a commencing epidemic, and something like this could happen to the VoIP networks in the future. It inspired us to propose SPIT structures for the network protection space. The work is performed using a modern voiceover-over-IP (VoIP) toolkit in the lab, and the devices there are used for other research ventures. The proposed design checks on the connections in its network such that any proxy server on the system would monitor the network would act as a buffer between other proxies and clients.

Naboulsi, D. et al. [6] presented a method to categorize network usages on the migratory population. We used a large-scale dataset in Abid, Ivory Coast, for our framework. These findings prove the framework's ability to classify calling profiles that rely on the measurement technique. However, it also effectively detects traffic profiles that are out of interest to discover.

Vieira, M. R. et al. [8] under their ubiquity, the social dynamics that they open, infrastructures have increased our research capabilities, particularly regarding city technology. The usage of these modern innovations may complement conventional methods. Various study groups have made various attempts to pinpointing hard-to-to-identify regions a priority. However, so far, the methods have suffered from some disadvantages, such as human-sized datasets, such as the failure to account for spatial resolution accurately.

\section{System Model}

Given an initial set of $\mathrm{TFD}=\left\{\mathrm{bts}_{1}\right.$; bts $2, . .$, btsn $\}$ that gives coverage to a geographical region $\mathrm{R}$ characterized by its Voronoi tessellation $\mathrm{R}=\left\{\mathrm{V}_{1} \mathrm{U} \mathrm{V}_{2} \mathrm{U} \ldots \mathrm{U} \mathrm{V}_{\mathrm{n}}\right\}$, we seek to discover the optimal disjoint subsets of TFD that cover areas within $\mathrm{R}$ where either the number of activities or unique users reach a maximum in a specific period t. An exhaustive exploration of all possible disjoint subsets of TFD becomes a daunting task as the number of TFD increases. Datasets are collected in real-world datasets.

\subsection{Call Data Record}

Every call to the service is recorded in a call-storing system. Identifies the caller, who received the fax, where it was sent, and who it was sent. There are no theoretical limits on how many Call detail Record (CDR) s the warehouse will hold. Additionally, old data could cease to be significant. Because of this, we use a sliding window to gather our info. A window is displayed by Tk where $\mathrm{k}>=\mathrm{n}+1$ time units - inserting a new device and removing the oldest device yields a new windows. It depends on the service provider to specify the scale and amount of time units.

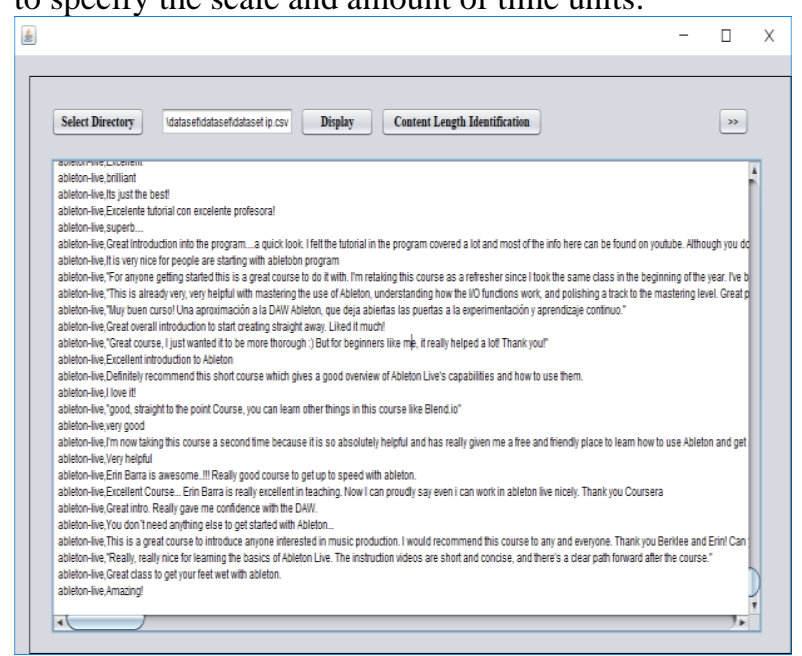

Figure 1: Dataset uploading process

Voice Spam Content: The substance of phone calls is either prerecorded human voice messages or computer text-to-to-speech programs. Telephone IVR functionality is also provided speech information utilizing a spam blocker. When the receiver answers an auto dialled request, they will talk or dial in with their computer. If there is an interaction, voice communication is played in its entirety. There are many common spam forms, such as telemarketing, election campaigns, debt recovery, and canvassing spam. We sourced 100 examples of publically accessible voicemail or voice recordings to help classify the audio in the telephone material. We do something to provide a public speech and voicemail interpretation. It is important to emphasize that, considering the non-random survey; these observations cannot be generalized to the voicemail receiver community's remainder. While this does include history and information on the voice mail, it's still needed to provide a picture of the whole campaign. We can use the following different spam forms, examples of which are most prevalent: credit card authentication, bogus taxes, and political quibbling. 


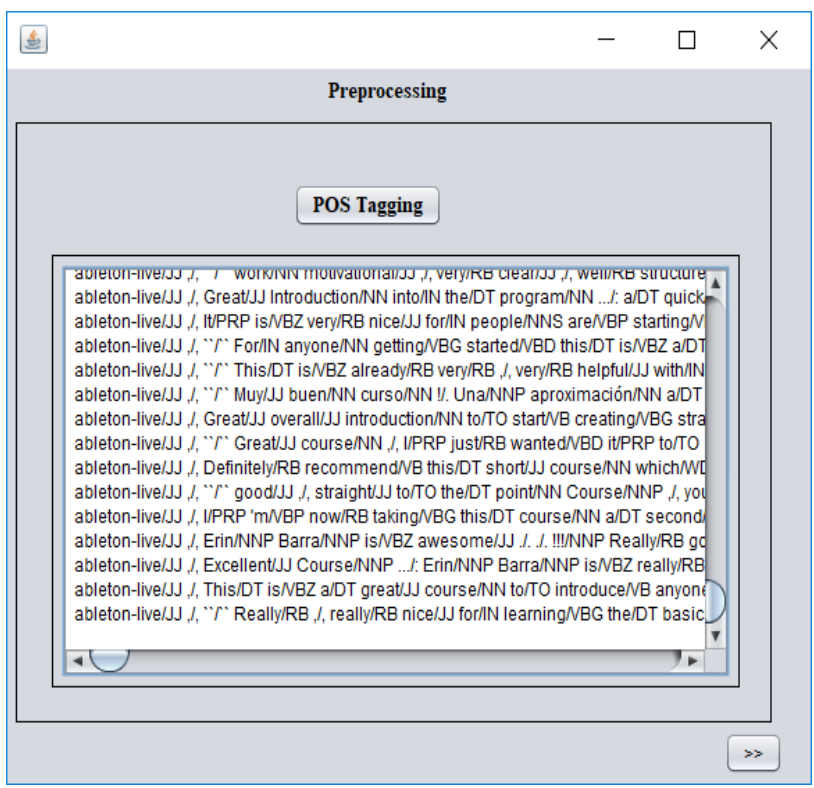

Figure 2: PoS Tagging Applying

When people get these fraudulent credit card calls, they are told that their card has been cancelled. To get their identification verified, they must have their credit card and social security number over the cell. When we only could hear the speech, the scammers spoofed the phone number to confuse us into thinking that it was the credit card firm.

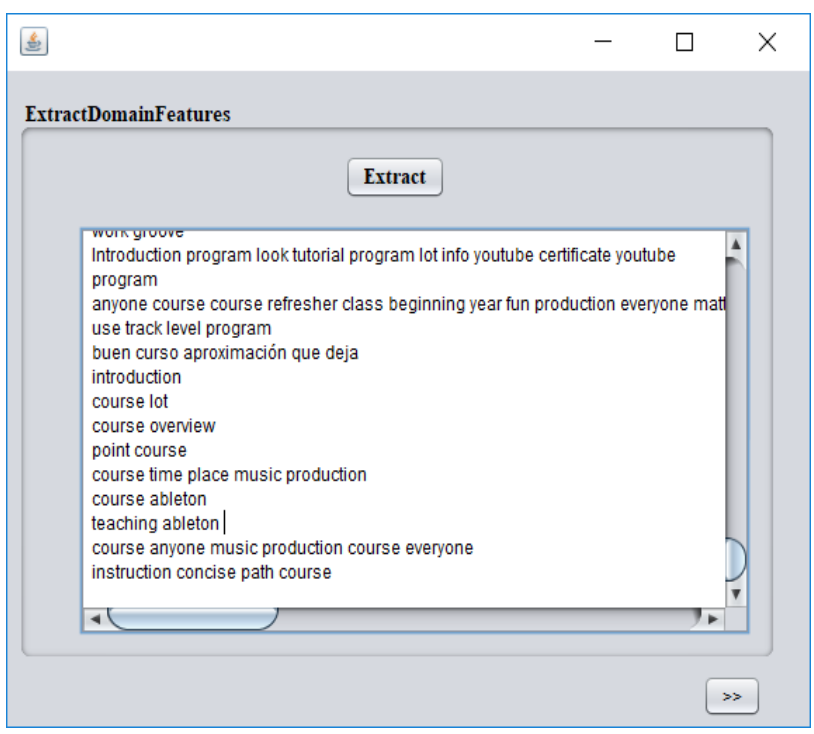

Figure 3: Extraction Process

This automated phone abuse technique had a voice-activated Digital Voice Message Device (VoAS) used to elicit and retrieve credit card details from victims.

The agents get a call describing themselves as an (Internal Revenue Service) IRS tax investigator and gives false credentials. She tells the receiver that they have to pay the IRS a certain fee. The demand is often followed by warnings of arrest or loss of a driver's license or a driving license if the money is not given quickly.

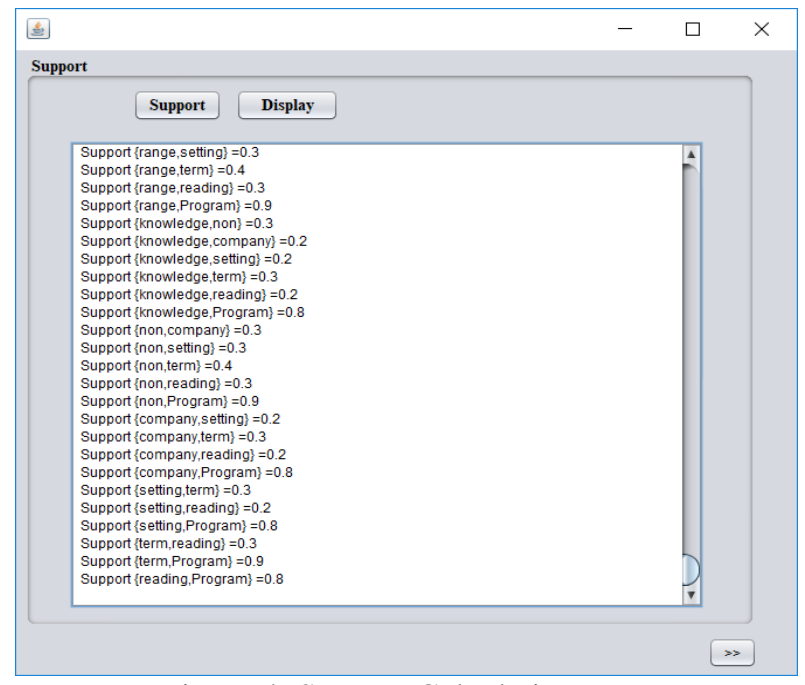

Figure 4: Support Calculation

\subsection{Nuisance Call Model}

They have labeled "nuisance" or "spam" calls because they are created without a consumer's express prior permission on a public phone network. It is also understood that telemarketing calls are used to benefit from selling and promoting or promotional purposes. On the other side, they can even be used in manipulative promotions that assist in phishing and theft. This portion contains a nuisance call classification, characteristics, and hazard model-nuisances in terms of calls automated phone calls may be created or managed by nuisance calls. Nuisance calls may be described as such: annoyance, impersonation, unsolicited sales, identify theft, and telemarketing.

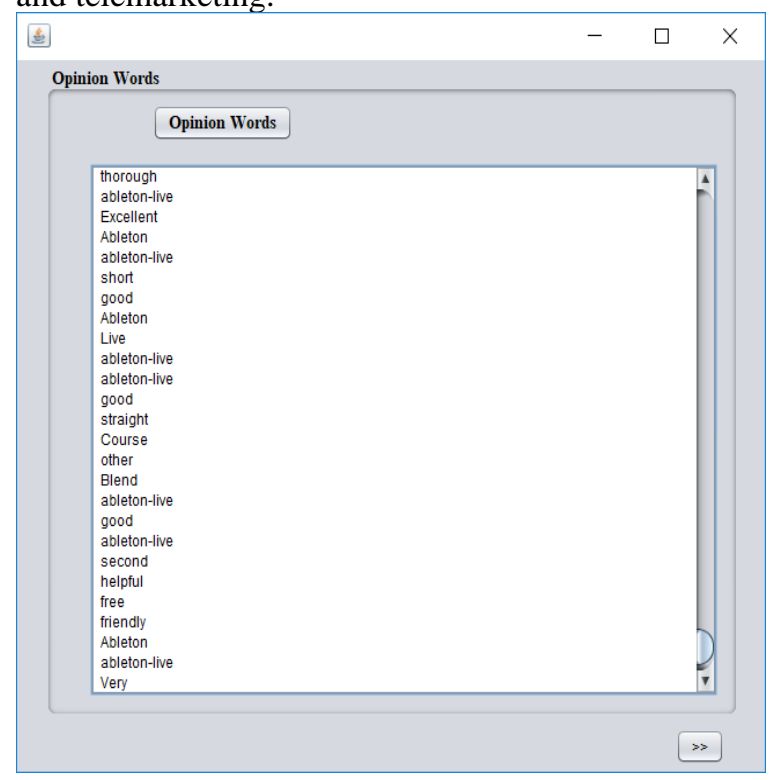

Figure 5: Opinion words Calculation

1. Telemarketing: The purpose of telemarketing is to reassure consumers of their purchase.

Phishing is used to perpetrate theft, such as credit card. Computer-dialed telephone calls are also regarded as reported nuisance calls. 


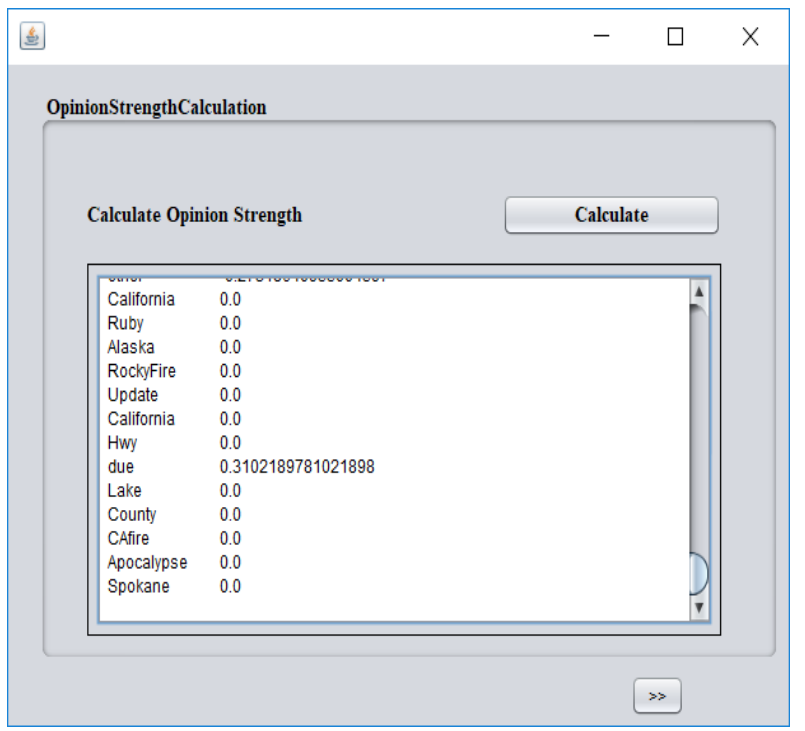

Figure 6: Opinion Strength Calculation

2. Distracting: Answering machines that annoy the recipients by generating "Distress" calls can be used to launch a Distributed Denial of Service (DDoS) attacks.

3. Spammers tend to be innovative, flexible, irreverent, reckless, well-intentioned, and able to adapt to situations, enthusiastic, but have high personal costs, and feel comfortable spreading gossip

4. In a sense, the spammer's primary goal is to obtain calls and business, sales and scam and phishing. This causes advanced behavior making them stick out in sharp comparison in the network: 5. Combinations of various calls seek to apply to multiple consumers, many of whom struggle to maintain the same degree of performance

6. The difference between non-reciprocal actions is that spammers seldom get calls from people who chat with them.

7. The spammer has a high number of outbound calls and a few inbound ones. Once the call is identified as spam, the normal called hangs up.

8. Higher than expected call volume: Spammers tend to call as many numbers as possible in a matter of time.

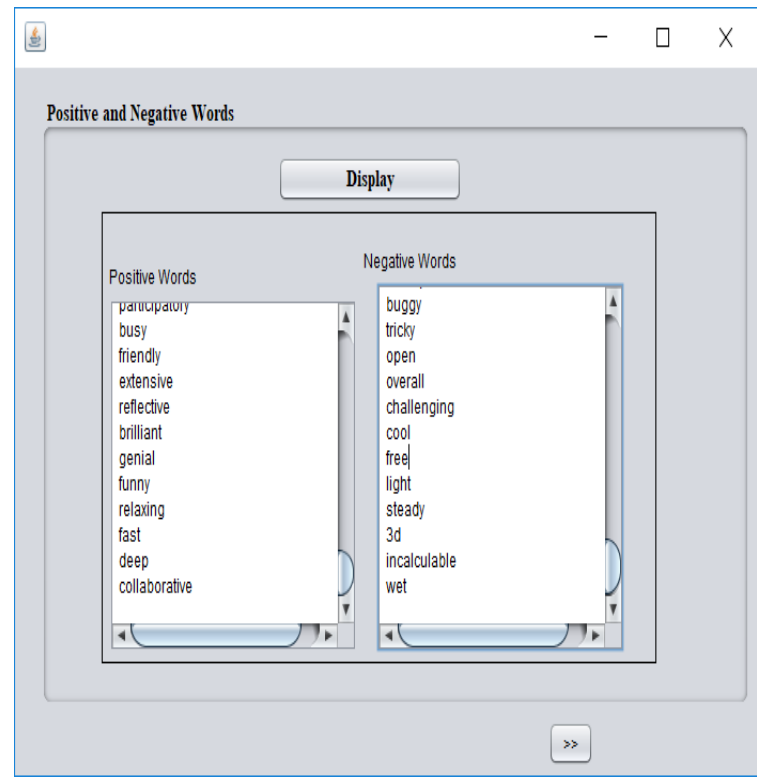

Figure 7: Displaying Positive and negative Words

3.3 CWRA: Consolidated Weighted Reputation Algorithm

Input:

F: Feature set (Spatial feature, temporal feature)

$\mathrm{G}$ : Grid set $\left(\mathrm{G}=\left(\left\{\mathrm{g}_{\mathrm{i}} \mathrm{j}\right\} g_{i=1, j=1}^{i=100, j=100}\right.\right.$ Where $\mathrm{g}_{\mathrm{i}} \mathrm{j}$ represents a single grid element in $100 \times 100$ Milan grid),

$\mathrm{A}_{\mathrm{CDR}}$ : Activity data of network cells belong to grid element $g_{\mathrm{i}} \mathrm{j}$,

$\mathrm{k}$ : No. of clusters

$\mathrm{C}_{\mathrm{k}=1-\mathrm{N}}$ : Cluster categories according to activity data

\section{Output:}

$\mathrm{C}_{\mathrm{t}}$ : List of optimized resource allocations for the cells in the grid

1 Network Resource Allocation (F, $A_{C D R}, G$ )

2 while cells from Grid set $\mathrm{G}$ not in k clusters do

$3 \mathrm{C}_{\mathrm{p}}=$ Predict cluster memberships for cells in Grid set $\mathrm{G}$

$4 \quad$ Allocate Network resources to each cell in $\mathrm{G}$ according to cluster membership and

5 end assign it to Optimized Set $\mathrm{C}_{\mathrm{t}}$

6 return $C_{t}$ 


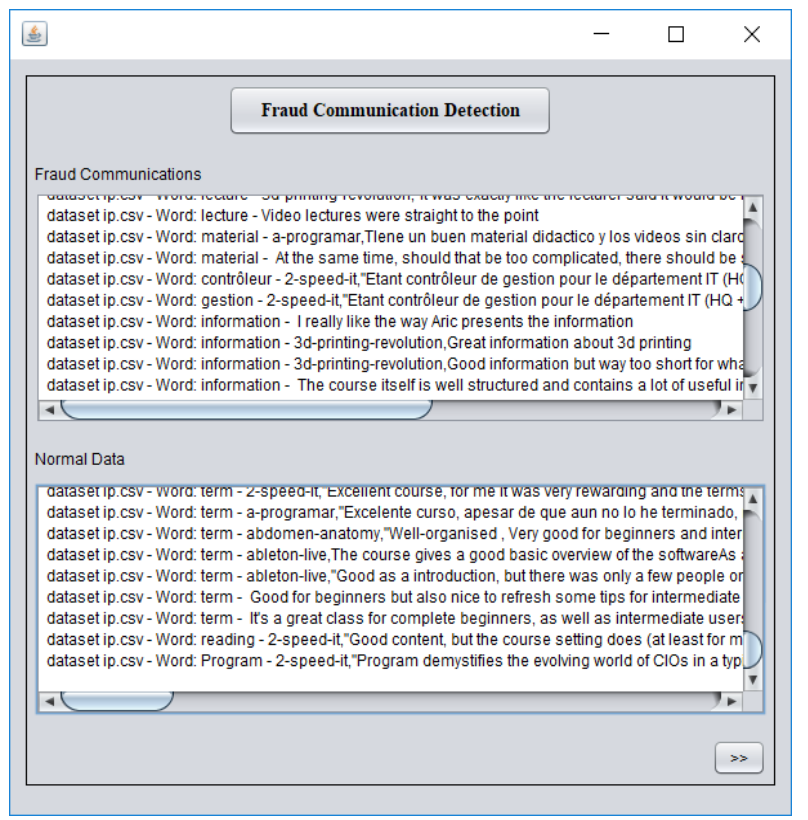

Figure 8: Fraud communication classification using the Naive Bayes algorithm

\subsection{Threat model:}

Various types of risks and assaults must be considered when designing a comprehensive countermeasure:

They were exchanging a current user's email address with a separate one that has not yet been decided to be connected to all others and then re-using it to begin the process of sending spam. If they are detected, spammers will exhibit very diverse patterns of behavior. Information can be passed around by spammers, even though it is true.

\section{Discussion}

The proposed model has implemented by using the java programming language. The proposed model compared by exiting authors.

\subsection{KEY CHALLENGES}

Fighting email spam is somewhat simpler; however, it does not solve the technological problems specific to phone calls.

\section{A. Simultaneity of Impatience}

As with text, a voice call would typically have an immediacy restriction. When a telephone call comes in, you will handle it as an emergency. Delays must be held to a minimum to keep the phone spam detection system successful. A wait will inconvenience the genuine callers and force them to hang up.

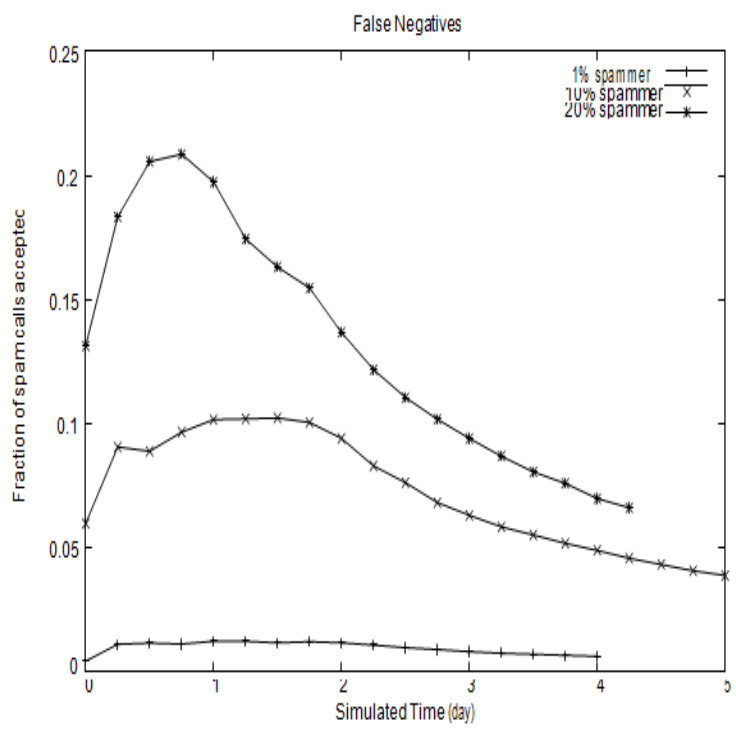

Figure 9: Effect of spammers

\section{B. problems: Audio streams are difficult}

A phone call's content can be challenging to parse and analyze: it is the audio of an email rather than a text message. Making it worse, the voice call only reveals the caller's message content when it is answered, so a spam system can disclose the message. A spam-block scheme is simple to decipher, which does not harm either the sender or the user.

\section{With limited or uninteresting header data}

Telephone calls miss the active topic and message details of a text. When a call arrives at the final destination, the details have been greatly compressed. Table III in the Appendix offers an illustration of an often encountered call header. Before including some text, an email header has well-defined and information-rich SMTP headers. Removing the sender's IP address and the domain name is a huge obstacle. The closely contrasted to a callable header, which decreases the risk of being used for spam.

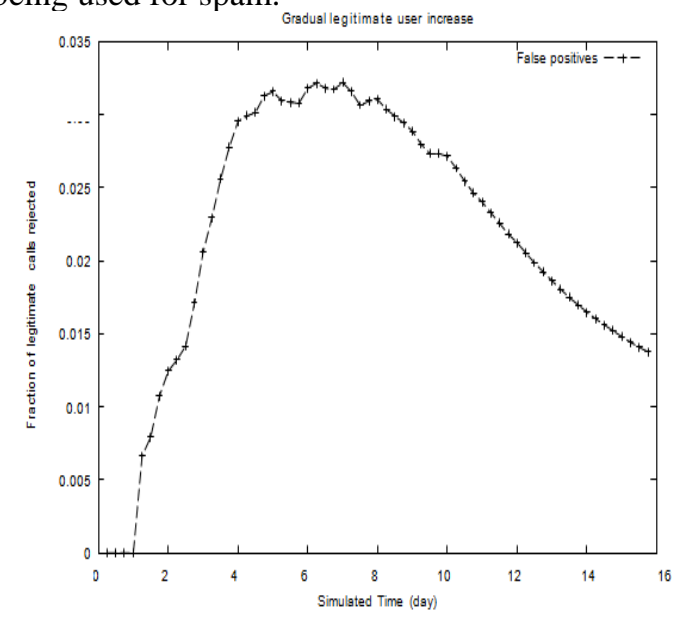

Figure 10: Adding legitimate users

\section{Challenging to gain goodwill}

Of course, you would have to ask your users for permission before installing a phone anti-spam 
system in their voicemail. Consumers have historically shown poor toleration for false positives. Email is less likely to be disabled, so when it is, the effects could be catastrophic.

\section{E. fictive caller ID}

It helps the receiver to determine who is calling until they answer the door. But caller ID doesn't authenticate, making it very vulnerable to being spoofed. To ensure the confidentiality of the call, the caller ID is provided by the TSP. If the spammer has subscribed to a TSP that permits caller ID customization, this protection is breached. Until recently, modifying caller ID was very costly for individuals and small companies (a newly acquired ISDN PRI trunk line will cost over $\$ 1,000$ per month and an internal telephone device will cost thousands of dollars). To this day, inexpensive and efficient caller ID spoofing over the Internet telephony features such as caller ID, spoofing Caller ID is child's play. It follows, then, that every email security strategy that depends on caller ID is now put at risk of being beaten by caller ID spoofing.

\section{F. The downside to SPAM is that the calls are more difficult to trace.}

Another way to deal with spam is to enact legislation that renders it a felony and then sees that offenders are punished. A limited number of users made up the bulk of the spammers' goals, causing large spam volume losses. On the other hand, closing down the stock botnet lowered unwanted global emails by $40 \%$. A similar spread of telephone scam artists is often to be assumed. Owing to the technological and legal difficulties in distinguishing real PSTN traffic and caller ID spoofing, it is impossible to distribute information on the distribution of telemarketers and smarthooders.

\section{Conclusion}

In this article, the TFD (data filtering) approach was suggested using the Consolidated Weighted-based Reputation (CW-R) algorithm. Therefore, there is no common consensus about how to tackle spam's problem by review and examination of current solutions. Designs so far come with tradeoffs: nice versus deployability versus protection. We think usability is the most critical criteria by looking at the whole landscape and strategies used in spam protection. Telephony is bound to lose any or fail, while email does not. We assume that callers won't put undue pressure on the receiver, and recipients won't put themselves at risk. Thus, prospective studies would understand both the caller's and the victim's application of the latest strategy from two separate viewpoints. One way we think we can thrive is by innovative usage of diverse strategies. Each technique has its advantages, which can be a combined technique. However, because the telephony method is real-time, the design does not add more difficulty. We found that utilizing two standalone approaches was the optimum combination of usability, flexibility, scalability, and reliability. Whenever we try to evaluate spam telemarketing, one problematic element still reoccurs in ID spoofing. We think that countering spam can be achieved with trustworthy and unmodified caller ID and recognizing improvements made that are minor. From our proposed Call Attributes methodology, we find it the most accessible and readily available. This, though, works against the robustness of the caller ID spoofing capabilities of the spammers. We believe that if caller ID spoofing were successfully thwarted, we believe that caller integrity analysis will answer.

\section{References}

[1] V. A. Balasubramaniyan, M. Ahamad, and H. Park, "Callrank: Combating spit using call duration, social networks and global reputation," in Fourth Conference on eamil and anti-spam (CEAS 2007), 2007

[2] H. Sengar, X. Wang, and A. Nichols, Call Behavioral Analysis to Thwart SPIT Attacks on VoIP Networks. Berlin, Heidelberg: Springer Berlin Heidelberg, 2012, pp. 501-510

[3] Cecaj, A., Mamei, M., \& Bicocchi, N. (2014). Re-identification of anonymized CDR datasets using social network data. 2014 IEEE International Conference on Pervasive Computing and Communication Workshops (PERCOM WORKSHOPS). doi:10.1109/percomw.2014.6815 210

[4] Javed, I., Toumi, K., \& Crespi, N. (2018). NCombat: A Nuisance Call Combating Framework for Internet Telephony. 2018 17th IEEE International Conference On Trust, Security And Privacy In Computing And Communications/ 12th IEEE International Conference On Big Data Science And Engineering (TrustCom/BigDataSE). doi:10.1109/trustcom/big datase.2018.00027

[5] Srihari, V., Kalpana, P., \& Anitha, R. (2014). Dendritic cell algorithm for preventing spam over IP telephony. 2014 International Conference on Informatics, Electronics \& Vision (ICIEV). doi:10.1109/iciev.2014.7135997

[6] Naboulsi, D., Stanica, R., \& Fiore, M. (2014). Classifying call profiles in large-scale mobile traffic datasets. IEEE INFOCOM 2014 IEEE Conference on Computer Communications. doi:10.1109/infocom.2014.6848 119

[7] Tu, H., Doupe, A., Zhao, Z., \& Ahn, G.-J. (2016). SoK: Everyone Hates Robocalls: A Survey of Techniques Against Telephone Spam. 2016 IEEE Symposium on Security and Privacy (SP). doi:10.1109/sp.2016.27

[8] Vieira, M. R., Frias-Martinez, V., Oliver, N., \& Frias-Martinez, E. (2010). Characterizing Dense Urban Areas from Mobile Phone-Call Data: Discovery and Social Dynamics. 2010 IEEE 
Second International Conference on Social Computing. doi:10.1109/socialcom.2010.41

[9] Xu, Q., Xiang, E. W., Yang, Q., Du, J., \& Zhong, J. (2012). SMS Spam Detection Using Noncontent Features. IEEE Intelligent Systems, 27(6), 44-51. doi:10.1109/mis.2012.3

[10]. A. Keromytis, "A comprehensive survey of voice over ip security research," IEEE Communications Surveys Tutorials, vol. 14, no. 2, pp. 514-537, Second 2012 